\title{
Capillary-Fiber Based Electrophoretic Delivery Device
}

David Poxson, Erik Gabrielsson, Alberto Bonisoli, Ulrika Linderhed, Tobias Abrahamsson, Isabelle Matthiesen, Klas Tybrandt, Magnus Berggren and Daniel Simon

The self-archived postprint version of this journal article is available at Linköping University Institutional Repository (DiVA):

http://urn.kb.se/resolve?urn=urn:nbn:se:liu:diva-157207

N.B.: When citing this work, cite the original publication.

Poxson, D., Gabrielsson, E., Bonisoli, A., Linderhed, U., Abrahamsson, T., Matthiesen, I., Tybrandt, K., Berggren, M., Simon, D., (2019), Capillary-Fiber Based Electrophoretic Delivery Device, ACS Applied Materials and Interfaces, 11(15), 14200-14207. https://doi.org/10.1021/acsami.8bo2268o

Original publication available at:

https://doi.org/10.1021/acsami.8b022680

Copyright: American Chemical Society

http://pubs.acs.org/ 


\section{Capillary fiber-based electrophoretic delivery device}

David J. Poxson ${ }^{a, \neq}$, Erik O. Gabrielsson ${ }^{a, \neq}$, Alberto Bonisolia,b,c, Ulrika Linderhed ${ }^{d, e}$, Tobias Abrahamsson $^{a, d}$, Isabelle Matthiesen ${ }^{a,}{ }^{+}$, Klas Tybrandt ${ }^{a}$, Magnus Berggren ${ }^{a,}{ }^{*}$, Daniel T. Simon $^{a}$

a) Laboratory of Organic Electronics, Department of Science and Technology, Linköping University, 60174 Norrköping, Sweden

b) Center for Micro-BioRobotics, Istituto Italiano di Tecnologia, 56025 Pontedera, Italy

c) BioRobotics Institute, Sant'Anna School of Advanced Studies, 56025 Pontedera, Italy

d) Department of Physics, Chemistry and Biology, Linköping University, 58183 Linköping, Sweden

e) Department of Printed Electronics, RISE Acreo, Research Institutes of Sweden, Norrköping, Sweden

Keywords: Electrophoresis, polyelectrolyte, iontronics, hyperbranched polymer, bioelectronics, substance delivery

Abstract

Organic electronic ion pumps (OEIPs) are a versatile tool for electrophoretic delivery of substances with high spatiotemporal resolution. To date, OEIPs and similar iontronic components have been fabricated using thin-film techniques, and often rely on laborious, multistep photolithographic processes. OEIPs have been demonstrated in a variety of in vitro and in vivo settings for controlling biological systems, but the thin-film form factor and limited repertoire of polyelectrolyte materials and device fabrication techniques unnecessarily constrain the possibilities for miniaturization and extremely localized substance delivery, e.g., the greater 
range of pharmaceutical compounds, on the scale of a single cell. Here, we demonstrate an entirely new OEIP form factor based on capillary fibers that include hyperbranched polyglycerols (dPGs) as the selective electrophoretic membrane. The dPGs enable electrophoretic channels with high concentration of fixed charges, well-controlled cross-linking, and can be realized using a simple "one-pot" fluidic manufacturing protocol. Selective electrophoretic transport of cations and anions of various sizes is demonstrated, including "large" substances difficult to transport with other OEIP technologies. We present a method for tailoring and characterizing the electrophoretic channels' fixed charge concentration in the operational state. Subsequently, we compare the experimental performance of these capillary-OEIPs to a computational model and are able to explain unexpected features in the ionic current for the transport and delivery of larger, lower mobility ionic compounds. From the model, we are able to elucidate several operational and design principles relevant for miniaturized electrophoretic drug delivery technologies in general. Overall, the compactness of the capillary-OEIP enables electrophoretic delivery devices with probe-like geometries, suitable for a variety of ionic compounds, paving the way for less-invasive implantation into biological systems and for healthcare applications.

\section{Introduction}

Organic bioelectronics ${ }^{1-3}$ is an emerging technology platform which incorporates the properties of biologically relevant organic materials with that of organic electronic devices to enable translation of signals across the biology-technology gap. Organic electronic materials can possess many of the archetypical bio-compatible, -mimetic, and -stable properties of polymer biomaterials in combination with biochemical-to-electronic (and converse) signal transduction properties, making them well-suited for a range of biological applications. Based on these favorable properties, various organic bioelectronic sensors ${ }^{4-6}$ and actuators ${ }^{7-10}$ have been developed and explored, both in vitro and in vivo, for various diagnostic, monitoring, and therapy applications. Another class of bioelectronic, electronic-to-biochemical translation device, is the so-called organic electronic ion pump ${ }^{11,12}$ (OEIP), an ionic delivery device based on electrophoretic transport through ionic charge selective membranes. 
Over the last decade, OEIPs and other iontronic devices have typically been manufactured using photolithographic processes yielding miniaturized devices with micron-sized features ${ }^{12}$ on planar substrates or as stand-alone thin films ${ }^{13,14}$, to achieve highly local and fast ${ }^{15}$ delivery of small charged molecules, e.g. alkali ions and neurotransmitters. These electrophoretic devices have been used to modulate neuronal cell and tissue functions using flow-free delivery of small biological signaling compounds at high spatiotemporal resolution, in vitro ${ }^{11,12}$, in vivo ${ }^{13,14,16}$, and recently in plants ${ }^{17}$.

Considering medical devices and technologies from the end-use perspective, capillaries, catheters, and fibers have proven to be versatile form factors in healthcare and medical research and are widely used in diagnostic, therapeutic, and surgical procedures. For guiding optical wavelengths, optical glass fibers have found their way into the areas of imaging, minimally invasive surgery, tissue ablation, biological sensing, and tissue diagnosis ${ }^{18}$. Catheters and capillaries are commonly utilized and explored to contain or guide liquids or to deliver dissolved materials or pressure changes to the hollow core outlet. Example applications include capillary electrophoresis ${ }^{19}$, balloon valvotomy ${ }^{20}$, and biocatalysis ${ }^{21}$. As such, here we fabricate and investigate devices combining the OEIP capabilities of selective electrophoretic substance delivery with the geometrical and usability advantages of glass capillary fibers, simultaneously serving as both encapsulation and substrate. By filling the hollow core $(20-50 \mu \mathrm{m}$ inner diameter), of the glass fibers with polyelectrolyte (PE), it is possible to readily create freestanding OEIP devices with probe-like geometries with an outlet on the scale of a single cell.

OEIP devices have typically been fabricated from anionic or cationic PE membranes that enable selective transport of cations or anions, respectively ${ }^{22}$. Charge selectivity of a PE is accomplished when the fixed charge concentration within the PE is significantly higher than the surrounding electrolyte. Owing to the charge selective transport of ions through a PE, chemical gradients of ions at specific locations and at specific times can be directly controlled by application of an electric field across the OEIP channel ${ }^{23}$. Previous demonstrations of OEIP technology have typically been made utilizing cross-linked semirandom networks of linear PEs, such as poly(styrenesulfonate) or poly(vinylbenzylchloride ${ }^{24}$. However, poly(styrenesulfonate) 
or poly(vinylbenzylchloride) PEs have failed to demonstrate the capability to transport and deliver larger ions or rigid ions comprising cyclic or aromatic structures.

As an alternative to linear PEs, here we utilize functionalized dendritic, hyperbranched polyglycerols (dPGs) to fabricate electrophoretic channels we refer to as "dendrolytes" (DLs). We utilize the previously reported cation functionalized dPGs (CdPG) for the transport of anions ${ }^{17}$, and additionally, we newly introduce an anion functionalized dPG analog (AdPG) for the transport of cations. The DLs enable the transport and delivery of relatively larger and more rigid ionic compounds, as compared to the cross-linked PEs in previously reported OEIPs. Indeed, delivery of aromatic compounds through CdPG-based OEIPs was recently demonstrated ${ }^{17}$. However, in those experiments, the observed ionic delivery was significantly lower than the applied electric charge. This large discrepancy between applied electronic and observed ionic currents led us to further investigate the transport phenomena of low-mobility ions in these DL-based capillaryOEIP devices.

Further, using the capillary-OEIP as a model device, we present techniques to characterize the key iontronic material parameters of OEIP devices, namely, the fixed charge concentration and ionic conductivity of PEs in the operational state. Additionally, by comparing experimental measurements of capillary-OEIPs with a computational physical model, we establish important general operational and design principles for OEIP devices. Specifically, through analysis of the results from our computational model we are able to elucidate critical relationships between the transported molecular species and the operating properties of the OEIP, i.e., the electrophoretic channel geometry, conductivity, and transport efficiency for a specific ion. 
(a)

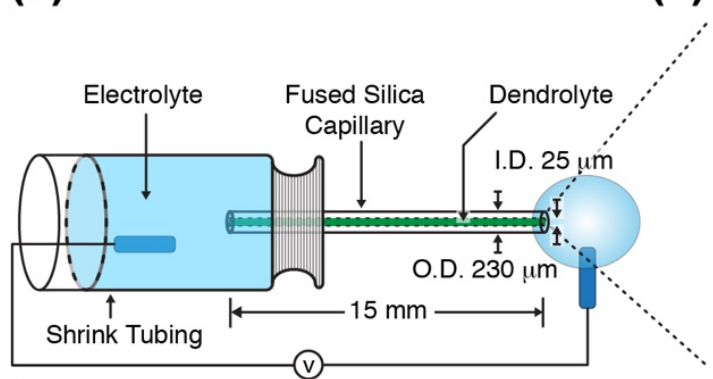

(b)

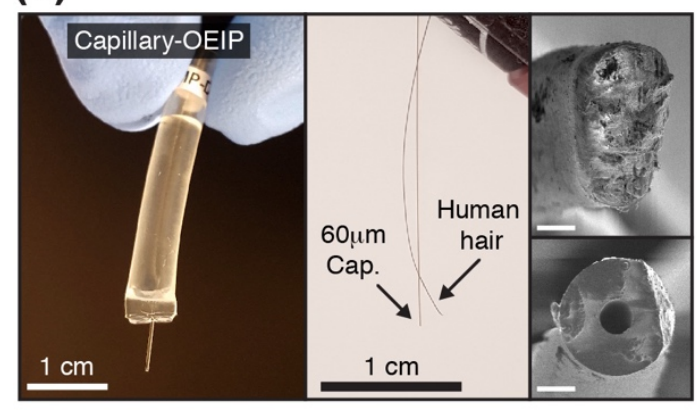

(c)

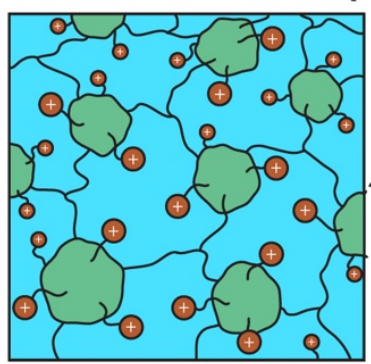

$50 \mathrm{wt} \%$

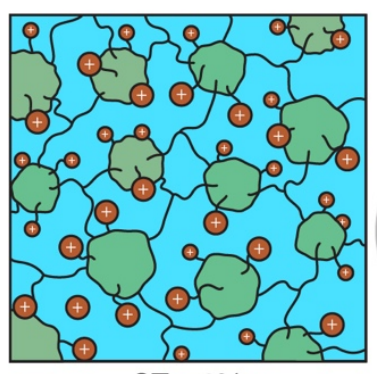

$67 \mathrm{wt} \%$ (d)
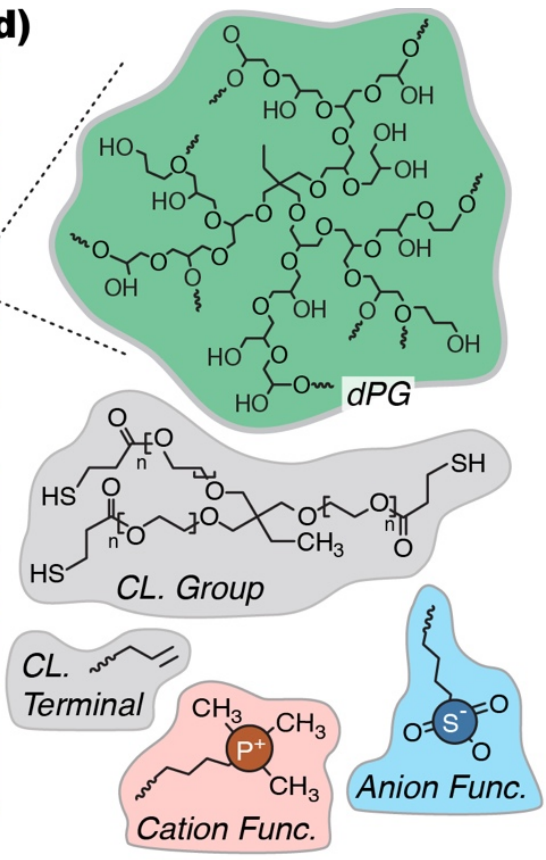

Figure 1. Capillary fiber based organic electronic ion pump. a) Schematic of OEIPs fabricated with the capillary fiber form factor. b) Images of fully fabricated capillary-OEIP, comparison of $60 \mu \mathrm{m}$ diameter capillary fiber to human hair, and cross sections of $60 \mu \mathrm{m}$ diameter capillary fiber and human hair (scale bar $20 \mu \mathrm{m}$ ). c) Conceptual diagram of dendrolyte based cation PE cross-linked networks at different weight percentage (50 and 67 wt\%). Crosslinking of DL at lower wt\% results in lower fixed charge concentration but higher nanoporosity. d) Chemical structures of dendrolyte constituent components: hyperbranched polyglycerol (green), allyl crosslinking group and thiol-based cross-linker (grey), anionic sulfonate functionalization (blue), and cationic trimethylphosphonium functionalization (red).

\section{Capillary-OEIP Fabrication}

A conceptual drawing of the DL material system along with the chemical structures for each of the DL's constituent components are illustrated in Figure $1 \mathrm{c}, \mathrm{d}$. The starting material in the synthesis of the DL based PE membranes was hyperbranched dendritic polyglycerol (dPG) with an average $M_{w}$ of $10 \mathrm{kDa}$ (degree of branching $\sim 53.6 \%$ and a mean number of 135 hydroxyl end groups) (Supporting information, Fig. S1). For newly synthesized AdPG, the hydroxyl end groups 
of the dPG were functionalized with approximately $20 \%$ ally/ groups, whose functional purpose was later utilized in thiol-ene crosslinking (Fig. 1d, grey). AdPG was obtained by deprotonation of the remaining hydroxyl groups ( $\sim 8 \%$ ), followed by nucleophilic substitution (in a ring opening mechanism of 1,4-butane sultone) to yield negatively charged sulfonate groups (Fig. 1d, blue). . Further details can be found in Supporting Information: Materials Synthesis and Characterization and Fig. S2. In the case of CdPG, previously synthesized cation functionalized dPG was used ${ }^{17}$.

Glass capillary fibers (fused silica) with a variety of dimensions, inner diameters (I.D.) of 20, 25 , and $50 \mu \mathrm{m}$, and outer diameters (O.D.), of 60,125 , and $230 \mu \mathrm{m}$ (protective polyimide coatings removed) were successfully utilized. In the experiments described here, unless otherwise noted, all data was taken with fibers I.D. $25 \mu \mathrm{m}$, and O.D. $230 \mu \mathrm{m}$ (Fig. 1a), owing to their good mechanical robustness.

To begin, the capillary fiber was cut to a length of $30 \mathrm{~cm}$ and the protective polyimide coating was removed thermally (550 ${ }^{\circ} \mathrm{C}$ for 1 hour) to facilitate UV cross-linking. To create an inner surface of the capillary fiber favorable for anchorage of the DL's, a combination of surface activation and adhesion promoter was used. The inner glass surface was first activated with hydroxyl groups by flushing the capillary fiber with $2 \mathrm{M}$ solution of $\mathrm{KOH}$ for 1 hour. Subsequently, epoxy groups were formed on the inner surface by flushing the capillary fiber with a $10 \%$ solution of the adhesion promoter 3-glycidoxypropyl trimethoxysilane (GOPS) in anhydrous toluene.

Immediately prior to fabrication of capillary-OEIPs, the stock DL (CdPG or AdPG) was dissolved in a solution of methanol, deionized water, and mixed with a thiol-based cross-linker (Thiocure ETTMP 1300) and a UV photo-initiator (Irgacure 2959) dissolved in methanol for a final weight 
percentage of DL at 50 or $67 \mathrm{wt} \%$. The capillary fiber was then filled with the DL solution until the solution could be seen emerging from the capillary outlet (after approximately $10 \mathrm{~min}$ ), and then placed under a UV lamp ( $254 \mathrm{~nm}, 2$ hours) for cross-linking. Finally, the capillary fiber was cleaved into individual sections, 7-15 mm in length, and immediately packaged with heat shrink tubing (serving as electrolyte reservoir), and stored in $10 \mathrm{mM}$ solution of $\mathrm{KCL}$. Further details for DL synthesis and capillary-OEIP fabrication are provided in Supporting Information: CapillaryOEIP fabrication. It is noted that the bare glass fibers used (particularly O.D.'s of 125 and $60 \mu \mathrm{m}$ ), in the millimeter lengths used, were relatively fragile to lateral strain and susceptible to breakage. Additionally, 125 and $60 \mu \mathrm{m}$ fibers were found to be suitable to readily pierce plant tissue (Fig. S6). Overall suitability for insertion or implantability of capillary-OEIPs is dictated by the application, but improvements to robustness of capillary-OEIPs could be made by preserving the protective polymer coating, or by using polymer based capillary fibers.

\section{Characterization}

The conductivity of an ion transported through the electrophoretic channel of the OEIP depends on the fundamental materials properties of the PE and its hydration state. Here, we compare the conductivity values of various anions and cations transported through capillaryOEIPs filled with CdPG and AdPG, respectively, made from solutions of different DL polymer concentrations (50 and 67 wt\%). In previous demonstrations of OEIP technology, the deposition of the monomers or polymers is made at lower concentrations, and the following polymerization or cross-linking of the PE occurs primarily in a mostly dry state, after which the PE is then 
rehydrated and allowed to swell within a semi-rigid encapsulation. By containing the PE inside capillaries or networks of capillaries ${ }^{25-28}$, owing to the low permeability to gases and liquids through the walls of the capillary, the concentration of solvent and solid DL content remains constant during the UV polymerization step (no undesired drying), and subsequently during exposure to electrolyte media (no reswelling). A significant source of error for estimating the hydrated PE volume is thus eliminated, enabling a reliable methodology to readily investigate PE iontronic parameters. Further, compared to previously published OEIPs, this capillary fabrication protocol enables a straightforward technique, starting from the constituent materials, to generate tailored OEIPs with homogenous channels with control over material characteristics of a PE membrane's nano-porosity, fixed charge concentration, and level of hydration.

Anions and cations were electrophoretically transported through CdPG and AdPG capillary-OEIPs from various $10 \mathrm{mM}$ aqueous source electrolytes to target solutions of $10 \mathrm{mM} \mathrm{KCl}$ (aq) until a steady-state electrical resistance was achieved. Anion/cation conductivities were then calculated for the capillary-OEIP channel of the specific OEIP geometry utilized (Table 1 and Table 2). The tabulated data indicates that ion conductivity and diffusivity values in the DLs compare well with other PE material systems ${ }^{29}$.

Table 1. Mean anion conductivities, $k$, with standard deviation $(n=8)$, and diffusion coefficients, $D_{i}$, in CdPG DL, with $\mathrm{K}^{+}$as co-ion.

\begin{tabular}{|c|c|c|c|c|}
\hline & \multicolumn{2}{|c|}{ CdPG 50 wt\% (0.76M) } & \multicolumn{2}{c|}{ CdPG 67 wt\% (1.07M) } \\
\hline & $k(\mathrm{~S} / \mathrm{m})$ & $D_{i}\left(\mathrm{~m}^{2} \mathrm{~s}^{-1}\right)$ & $k(\mathrm{~S} / \mathrm{m})$ & $D_{i}\left(\mathrm{~m}^{2} \mathrm{~s}^{-1}\right)$ \\
\hline $\mathrm{Cl}^{-}$ & $\mathbf{1 . 4 2} \pm 0.04$ & $\mathbf{5 . 0 1} \times 10^{-10}$ & $\mathbf{0 . 9 8} \pm 0.04$ & $\mathbf{2 . 4 5 \times 1 0 ^ { - 1 0 }}$ \\
\hline $\mathrm{I}^{-}$ & $\mathbf{0 . 8 6} \pm 0.03$ & $\mathbf{3 . 0 3} \times 10^{-10}$ & $\mathbf{0 . 5 3} \pm 0.03$ & $\mathbf{1 . 3 3} \times 10^{-10}$ \\
\hline
\end{tabular}




\begin{tabular}{|c|c|c|c|c|}
\hline $\mathrm{ClO}_{4}^{-}$ & $0.48 \pm 0.01$ & $1.69 \times 10^{-10}$ & $0.32 \pm 0.01$ & $8.01 \times 10^{-11}$ \\
\hline \multicolumn{5}{|c|}{$\begin{array}{l}\text { Table 2. Mean cation conductivities, } k \text {, with standard deviation }(\mathrm{n}=8) \text {, and diffusion } \\
\text { coefficients, } D_{i} \text {, in AdPG DL, with } \mathrm{Cl}^{-} \text {as co-ion. }\end{array}$} \\
\hline & \multicolumn{2}{|c|}{ AdPG 50 wt\% (1.21M) } & \multicolumn{2}{|c|}{ AdPG 67 wt\% (1.61M) } \\
\hline & $k(\mathrm{~S} / \mathrm{m})$ & $D_{i}\left(\mathrm{~m}^{2} \mathrm{~s}^{-1}\right)$ & $k(\mathrm{~S} / \mathrm{m})$ & $D_{i}\left(\mathrm{~m}^{2} \mathrm{~s}^{-1}\right)$ \\
\hline $\mathrm{K}^{+}$ & $3.12 \pm 0.10$ & $6.91 \times 10^{-10}$ & $2.79 \pm 0.13$ & $4.64 \times 10^{-10}$ \\
\hline $\mathrm{Na}^{+}$ & $2.03 \pm 0.10$ & $4.50 \times 10^{-10}$ & $1.79 \pm 0.07$ & $2.98 \times 10^{-10}$ \\
\hline $\mathrm{Li}^{+}$ & $1.38 \pm 0.11$ & $3.06 \times 10^{-10}$ & $1.23 \pm 0.06$ & $2.05 \times 10^{-10}$ \\
\hline Acetylcholine $^{+}$ & $0.91 \pm 0.05$ & $2.01 \times 10^{-10}$ & - & - \\
\hline
\end{tabular}

Estimating the concentration of fixed charges in a PE

For a membrane with a specific nano-scale structure and level of hydration, the concentration of fixed charges is a key physical parameter dictating ion transport characteristics. Other factors aside, the ionic conductivity of the PE channel typically increases with the fixed charge concentration. Further, the degree of selectivity towards transport of either cations or anions (permselectivity) is dependent on the PE's fixed charge concentration. To achieve permselectivity, the fixed charge concentration of the PE should be significantly higher than that of the surrounding electrolyte concentration ${ }^{23}$. Importantly, the permselectivity of a PE results in preferential unidirectional transport of desired ionic species and minimal transport of (oppositely charged) co-ions, thus enabling a strong correspondence between the applied electric current and the rate of delivery for the ion of interest. Therefore, it is important to determine the fixed charge concentration of a PE in its operational state. To this end, we present here a practical measurement method to estimate the fixed charge concentration of a PE using an ion exchange process in the electrophoretic transport channel of a capillary-OEIP device (Fig. 2). 


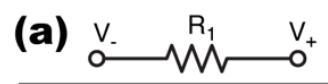

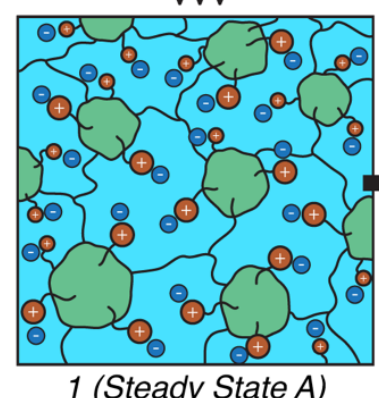

1 (Steady State A)

(b)

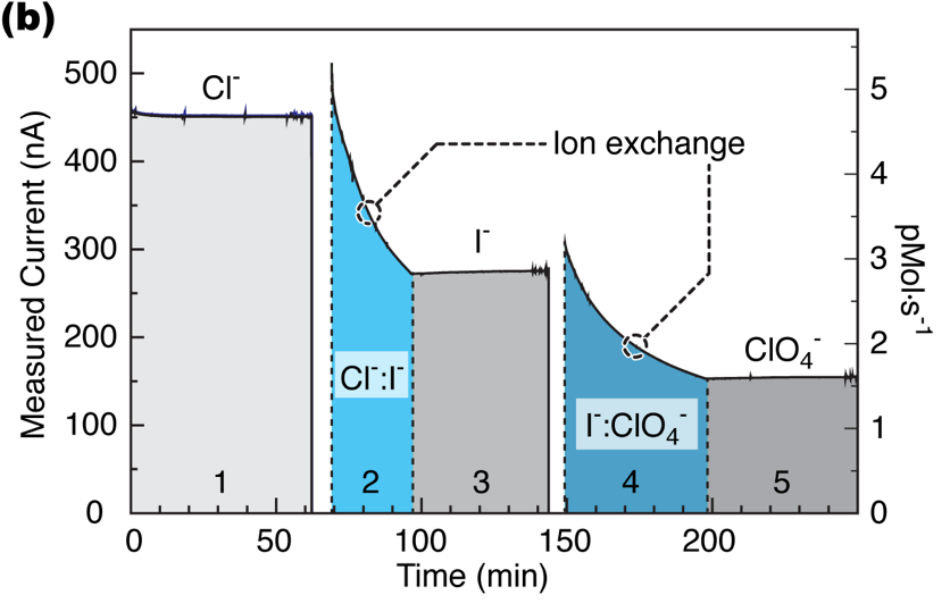

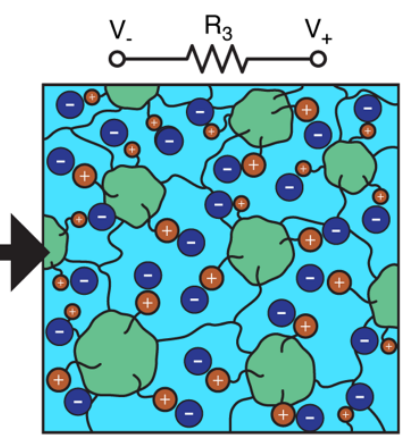

3 (Steady State B)

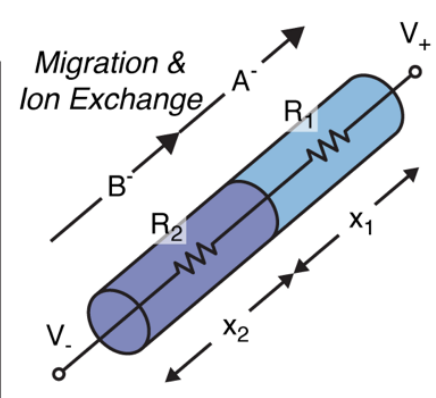

(c)

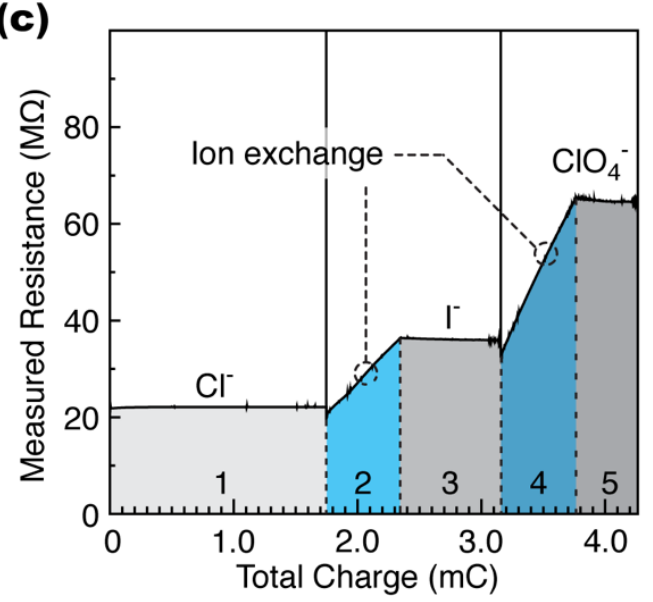

Figure 2. Ion exchange experiment to determine an OEIP PE's fixed charge concentration, each ion exchange step is numbered. a) The electrical resistance across a PE channel is proportional to the total ionic resistance of each ionic species contributing to electrophoresis (weighted according to number percentage in the PE channel). b) CdPG based capillary-OEIPs were operated at constant voltage while sequentially switching the source electrolyte from $\mathrm{Cl}^{-}$to $\mathrm{I}^{-}$to $\mathrm{ClO}_{4}^{-}$. By integrating the electronic current during ion exchange, a direct estimation of an OEIP PE's fixed charge concentration is made. c) Total resistance across capillary-OEIP plotted vs. total charge transported. Total resistance is the series resistance contribution of each ionic species.

Ion exchange experiments were performed on capillary-OEIPs filled with CdPG or AdPG DLs. The devices were operated at constant voltage while sequentially switching the source electrolyte from high-mobility ions to low-mobility ions (Fig. 2a). By integrating the resulting electronic current over the time interval the mobile ions inside the $\mathrm{DL}$ are exchanged through electrophoresis, a direct estimation of the fixed charge concentration of the DLs is made. 
The ion exchange measurement makes four assumptions: (i) local electroneutrality, the densities of the PEs' fixed charges and of the mobile transported ionic specie are equal inside the OEIP channel; (ii) through Donnan exclusion, co-ions are completely excluded from the channel, and thus do not contribute to the total ionic current; (iii) there are no other sources of current, such as chemical reactions in the electrolyte, PE, or at their boundary (e.g. water splitting); (iv) there is no unintended transport of lower mobility ions before higher mobility ions (all higher mobility ions leave the channel first). To test the validity of assumption (ii), the charge selectivity of AdPG and CdPG capillary-OEIPs was demonstrated by varying the target electrolyte $(\mathrm{KCl})$ concentration from $1 \mathrm{mM}$ to $1 \mathrm{M}$ during operation. It was found that selectivity of both AdPG and CdPG PEs is maintained at, or below, target electrolyte concentrations of $100 \mathrm{mM}$. Details of these experiments are provided in Supporting Information: dPG selectivity measurement, and Fig. S5. Further, to increase the accuracy of the ion exchange measurement, the ion exchange experiments were performed using electrolyte concentration of $10 \mathrm{mM}$ in order to be well below the selectivity limit for these PEs.

The electrophoresis driving ion exchange can be divided into three steps (Fig. 2). During step 1, the OEIP is operated with ionic species $A\left(\mathrm{Cl}^{-}\right)$, each fixed charge group within the DL is compensated for with an ion of species $\left.\mathrm{A}_{(\mathrm{Cl}}{ }^{-}\right)$, and OEIP channel resistance maintains a constant value. In step 2, source electrolyte A is switched for a lower mobility ionic species B $\left(I^{-}\right)$. During step 2, as ions of species A leave the PE channel, the fixed charge sites of the PE are exchanged with ions of species B in a one-to-one fashion. The exchange of higher mobility species A with lower mobility species $B$ results in an increase in electrical resistance across the OEIP, and the total resistance is the linear combination of the relative percentage contribution of both species. As can be seen in Fig. 2, when all of the fixed charge sites in the channel are exchanged from A to $B$, the resistance of the channel comes to a new steady-state value (step 3).

Given the assumptions listed above, the total charge and corresponding number of ions exchanged in the PE in order to replace ion $A$ with ion $B$, can be estimated by integrating the electronic current, $I(t)$, between the two steady-state conditions of step 1 and 3 :

$$
\text { Total moles exchanged }=\frac{1}{z F} \int_{t_{i}}^{t_{f}} I(t) d t \quad \text { (Eq. 1) }
$$


where $z$ is the charge number of the transported ion, $F\left(96485 \mathrm{C} \mathrm{mol}^{-1}\right)$ is Faraday's constant, and $t_{i}$ and $t_{f}$ are the beginning and end times of the ionic exchange process, respectively. The molarity of the PE in the channel can then be estimated by dividing the number of ions exchanged calculated from Eq. 1 by the volume of the capillary-OEIP channel.

Using this method, CdPG DL channels prepared at 50 and $67 \mathrm{wt} \%$ in capillary-OEIPs were found to have fixed charge concentrations of $0.76 \pm 0.05 \mathrm{M}$, and $1.07 \pm 0.04 \mathrm{M}$, respectively, and AdPG filled DL channels prepared at 50 and $67 \mathrm{wt} \%$ in capillary-OEIPs were found to have fixed charge concentrations of $1.21 \pm 0.04 \mathrm{M}$, and $1.62 \pm 0.03 \mathrm{M}$, respectively. From the estimated fixed charge concentration values, and using the Nernst-Einstein equation, the diffusion coefficients are calculated from the measured ionic conductivity values of the DLs reported above (Tables $1 \& 2$ ). Thus, as expected, we find that the concentration of fixed charges increases with the concentration of DL in the channel. However, as evident from Tables 1 and 2, there is an apparent tradeoff between achieving a high ionic mobility and simultaneously a high fixed charge concentration. For both AdPG and CdPG, and for the ions studied, an increase of the fixed charge concentration actually results in lower ionic mobility. Apparently, increasing the density of the PE channel, and it's associated porous-structures, has a stronger impact on the ion conductivity than increasing the fixed charge concentration of the DL from 50 to $67 \mathrm{wt} \%$.

\section{Transport behavior of low mobility ions}

The transport of low diffusivity $\left(D_{i} \lesssim 10^{-10} \mathrm{~m}^{2} \mathrm{~s}^{-1}\right.$ ) charged molecules has proven difficult in OEIPs based on linear PEs, leading to low or negligible delivery for such substances ${ }^{17}$. This limitation encouraged us to investigate the DLs capability to transport larger and more rigid charged compounds utilizing the transparent and geometrically well constrained glass capillary form factor. To this end, we performed ion exchange experiments from $\mathrm{Cl}^{-}$to the anionic dye molecule indigo carmine ${ }^{2-}$ (inset Fig. 3a) in the CdPG based capillary-OEIPs under constant applied current of 25 nA. We observed the ion migration characteristics using optical microscopy while recording the voltage. As described above, as $\mathrm{Cl}^{-}$was exchanged to indigo carmine ${ }^{2-}$ in the $\mathrm{DL}$ channel, there was a corresponding measured linear increase in channel resistance (Fig 3a). Additionally, we were able to optically follow the progressive replacement of $\mathrm{Cl}^{-}$counterions with 
indigo carmine ${ }^{2-}$ in the DL (Supporting Information, Fig. S7.). During the ion exchange process, indigo carmine ${ }^{2-}$ filled the volume of DL in the capillary-OEIP in direct correspondence to the electronic driving current, time elapsed, and the previously estimated molarity of the CdPG DL from above. As the indigo carmine ${ }^{2-}$ reached the outlet of the capillary-OEIP, indicating the completion of the ion exchange process, the recorded voltage across the capillary-OEIP decreased rapidly (Fig. 3a). Further, regardless of the applied current levels to the capillary-OEIP, no visual evidence of indigo carmine ${ }^{2-}$ escaping out from the delivery tip could be made. Verification of large ion transport was made using UV absorption spectroscopy of the dye molecules indigo carmine and methylene blue, for electrolyte samples taken for extended duration experiments (hours). CdPG based devices were measured to have a $26 \%$ efficiency at $25 \mathrm{nA}$, and a 7\% efficiency at $5 \mathrm{nA}$. AdPG based devices were found to have a $6 \%$ efficiency at both 10 and $50 \mathrm{nA}$. Details for these experiments are provided in Supporting Information: Measurement of Large Ion Delivery. 
(a)

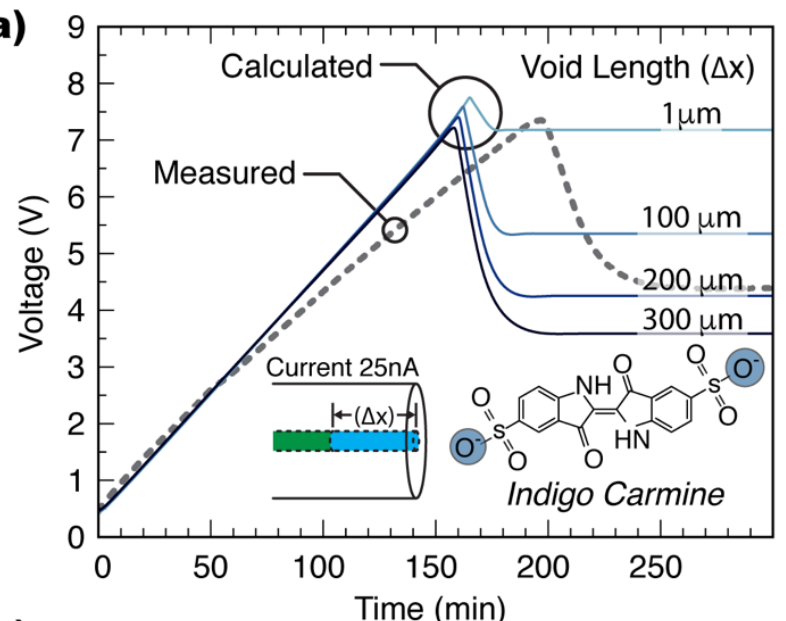

(c)

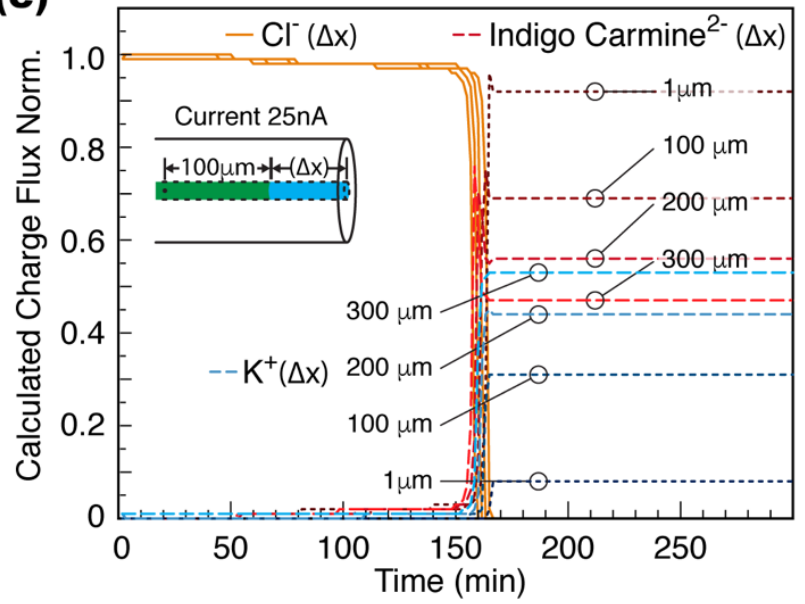

(b)

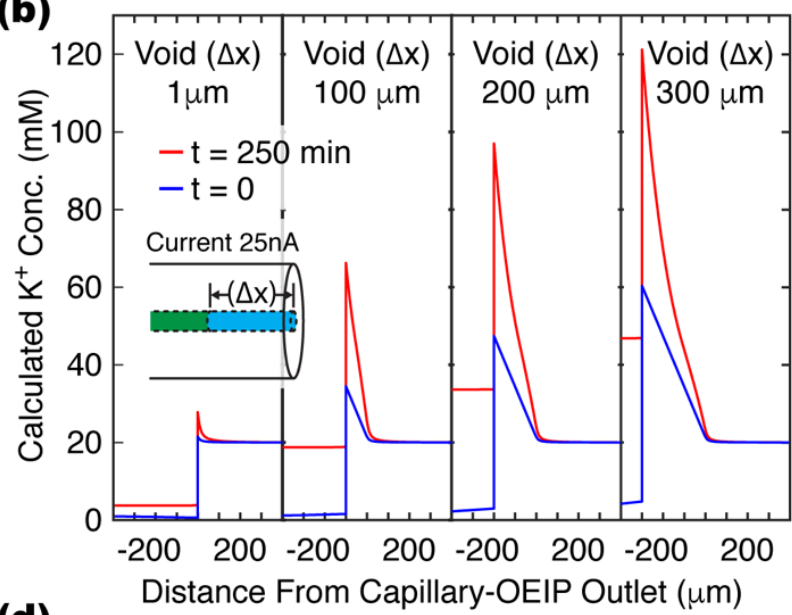

(d)

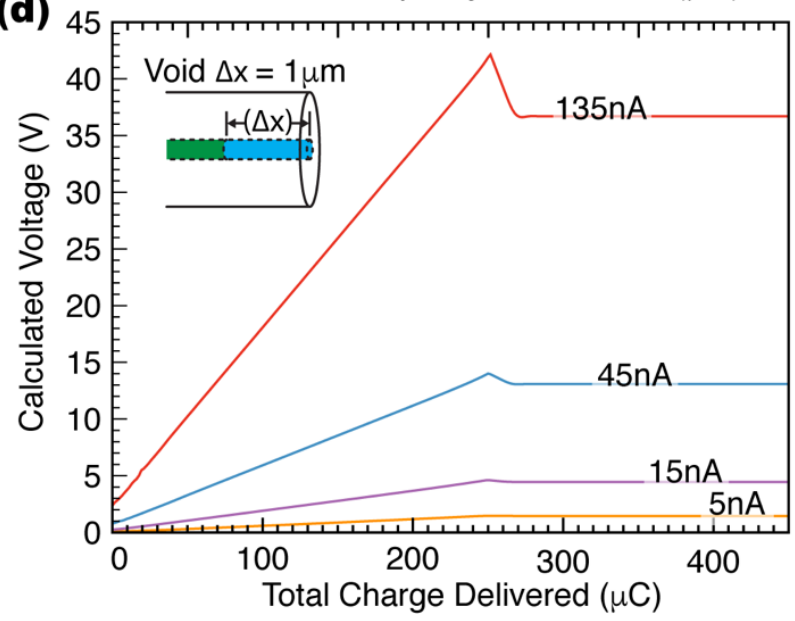

Figure 3. a) Measured and calculated voltage for a capillary-OEIP operated at $25 \mathrm{nA}$ during an ion exchange from $\mathrm{Cl}^{-}$to indigo carmine ${ }^{2-}$. The computational model incorporates the presence of a void in the PE, of length $\Delta x,(1,100,200$, and $300 \mu \mathrm{m})$ at the outlet of the capillaryOEIP (see inset), the anionic dye molecule indigo carmine ${ }^{2-}$ is shown in the inset. b) The calculated co-ion $\mathrm{K}^{+}$concentration across the capillary-OEIP for PE voids of length $\Delta \times(1,100$, 200, and $300 \mu \mathrm{m}$ ), before (blue) and after (red) ion exchange from $\mathrm{Cl}^{-}$to indigo carmine-, with an operating current of $25 \mathrm{nA}$. c) The normalized charge flux contribution of $\mathrm{Cl}^{-}$, indigo carmine ${ }^{2-}$ , and $\mathrm{K}^{+}$is calculated $100 \mu \mathrm{m}$ from the end of the PE (see inset) for voids of length $\Delta x(1,100$, 200, and $300 \mu \mathrm{m})$, during an ion exchange of $\mathrm{Cl}^{-}$to indigo carmine ${ }^{2-}$. d) The calculated voltage for operation at constant currents of 5, 15, 45 and 135 nA in a capillary-OEIP with a void length $\Delta \mathrm{x}$ of $1 \mu \mathrm{m}$. 
To investigate this unexpected low efficiency, and apparent operational voltage/current behavior, a computational model of the capillary-OEIP was developed using a finite element Nernst-Planck-Poisson model (see Supporting Information: Numerical Simulation for model details). ${ }^{30,31}$ The model implements the capillary-OEIP geometry and uses the experimentally determined values for the density of fixed charges of 50 wt\% CdPG (0.76 M). To fit the current levels recorded in the device, the $\mathrm{K}^{+}$and $\mathrm{Cl}^{-}$mobilities within the channel were reduced by a factor of 0.15 , which is in line with previous reports ${ }^{32,33}$, while the mobility of indigo carmine ${ }^{2-}$ was reduced by a factor of $0.96 .^{34}$ Importantly, two additional assumptions were made based on experimental observations. i) In the case that the DL channel does not extend all the way to the end of capillary-OEIP outlet (inset Fig. 3a), the remaining "void" at the end of the channel is assumed to be filled with the target electrolyte solution. ii), As indigo carmine has a limited solubility of $20 \mathrm{mM}$ in water, and because the channel comprises $50 \mathrm{wt} \%$ water, the solubility within the $\mathrm{DL}$ channel was assumed to be $10 \mathrm{mM}$, with the indigo carmine above $10 \mathrm{mM}$ precipitating from solution. Computational modeling was then performed for capillary-OEIPs with channel voids of various lengths ( $\Delta x=1,100,200$, and $300 \mu \mathrm{m}$; Fig. 3a). With a channel void of $200 \mu \mathrm{m}$, the model shows good agreement between the experimental voltage measurements of a capillary-OEIP operated at $25 \mathrm{nA}$ while exchanging $\mathrm{Cl}^{-}$to indigo carmine ${ }^{2-}$ in the $\mathrm{DL}$ channel (Fig. 3a).

The current and voltage characteristics of the capillary-OEIP can be understood by analyzing the changes in local ion concentrations at the channel outlet. Figure $3 \mathrm{~b}$ displays the values of the calculated $\mathrm{K}^{+}$co-ion concentration, across the capillary-OEIP outlet, for channel voids of various lengths $(\Delta x=1,100,200$, and $300 \mu \mathrm{m})$ during delivery of $\mathrm{Cl}^{-}$anions (blue, $\left.\mathrm{t}=0\right)$ as compared to delivery of indigo carmine ${ }^{2-}$ anions ( $r e d, t=250 \mathrm{~min}$ ). Because the transport of ions in an electrolyte next to a selective membrane is mainly diffusive in nature, the presence of ionic currents is strongly associated with local ion concentration gradients. The steepness of these ion concentration gradient depends on the mobility of the ionic species, and the total ionic current across the device. Thus, the concentration gradient of a highly mobile ion such as $\mathrm{Cl}^{-}$is less steep than the much lower mobility ion indigo carmine ${ }^{2-}$ for the same current (Fig. 3b). Therefore, the local increase in ion concentration at the PE/electrolyte interface is caused by the 
steepness of the ion concentration gradient, multiplied by its spatial extent. The increase in concentration gradient is more pronounced in a 1D diffusion geometry (void in the PE of a capillary-OEIP) compared to a 3D diffusion geometry (PE that terminates at the end of the capillary fiber).

Taken together, the local ion concentration at the PE/electrolyte interface increases as indigo carmine ${ }^{2-}$ reaches the outlet, which in turn causes the permselectivity of the membrane to decrease. This breakdown in permselectivity leads to a backflow of highly mobile $\mathrm{K}^{+}$ions. From the calculations, despite having a low relative concentration within the PE channel, $\mathrm{K}^{+}$backflow accounts for a significant portion of the overall current. This backflow of $\mathrm{K}^{+}$ions effectively increases the conductivity of the channel, which is seen as a drop in the operational voltage of the device, Fig. $3 a$.

The length of the void in the PE channel determines the spatial extent of the concentration gradient. Thus, a longer void will generate a higher local concentration of ions at the PE/electrolyte interface, and will result in greater backflow (Fig. 3a,b). The influence of void length on the permselectivity of the PE channel can be seen in Figure 3c, where the relative ionic fluxes in the capillary-OEIP channel are plotted for $\mathrm{Cl}^{-}$, indigo carmine ${ }^{2-}$, and $\mathrm{K}^{+}$during the $\mathrm{Cl}^{-}$-toindigo carmine ${ }^{2-}$ ion exchange process for channel voids of various lengths $(\Delta x=1,100,200$, and $300 \mu \mathrm{m})$. Initially, only $\mathrm{Cl}^{-}$is transported through the PE channel, but once the indigo carmine reaches the PE outlet, the backflow of $\mathrm{K}^{+}$increases significantly. From these calculation, it can be seen that even at the relatively low operational current level of $25 \mathrm{nA}$, for a sufficiently large void length $(200 \mu \mathrm{m})$, the majority of the overall current will comprise backflow of $\mathrm{K}^{+}$co-ions.

Another factor which potentially affects the permselectivity of the channel is the limited solubility of indigo carmine within the PE. To test the influence of the ion solubility within the PE on the model's results, two calculations were performed to fit the experimental data, one which took into account the limited solubility and one which neglects solubility limitations of indigo carmine. Interestingly, the two models show very similar characteristics (Fig. S8), suggesting that the void length in combination with ionic mobilities are the most important parameters of the system. Although it is unlikely that $380 \mathrm{mM}$ of indigo carmine would be soluble within the 
channel, the model does not distinguish between low solubility and low diffusion coefficient, as adjustments to either parameter result in similar calculated behavior. Additional studies are necessary to further elucidate the role that ion solubility within the PE plays on the operation of OEIP devices.

From the results of the model, the breakdown in selectivity arises due to the high ionic concentrations resulting from a concentration polarization in the channel void. Thus, the question arises if the concentration polarization effect can be avoided using lower device currents and a favorable channel void geometry. To investigate this possibility, we calculated the voltage across the capillary-OEIP channel with a fixed $1 \mu \mathrm{m}$ long channel void at various operational current levels $(5,15,45$, and $135 \mathrm{nA})$ during the ion exchange process, i.e. as $\mathrm{Cl}^{-}$is gradually replaced by indigo carmine ${ }^{2-}$. From Figure $3 d$, and comparing relative ionic flux contributions for indigo carmine ${ }^{2-}$ and $\mathrm{K}^{+}$, we conclude that the breakdown in selectivity scales with the applied current. By using lower delivery current, the amount of $\mathrm{K}^{+}$backflow can thus be reduced, resulting in improved pumping efficiency.

\section{Conclusion and Discussion}

We have demonstrated a fabrication methodology to produce miniaturized OEIP devices within a capillary fiber form factor. The capillary simplifies and reduces the overall size of the device structure by combining substrate and encapsulation layers, enabling OEIP devices with a probe-like geometry on the scale of human hair and with a delivery outlet on the scale of a single cell. Preliminary experiments suggest the suitability of these capillary-OEIPs to be useful for a variety of studies involving insertion into biological tissue.

These capillary-OEIP devices are based on a new class of PE: hyperbranched polyglycerols (dPGs) forming so-called dendrolytes (DLs), that enable the selective transport of cations and anions of various sizes, including larger and more rigid compounds. Additionally, thanks to the favorable solubility properties of the DLs, it is possible to apply solutions even up to 67 wt\% of DL in a "one-pot" three-component miscible mixture along with a cross-linker and UV photoinitiator to the capillary inlet before curing. This one-pot method provides a means for achieving membranes (loaded capillaries) with high solid content over extended lengths, even beyond 
centimeters. By varying DL concentration, an easy design parameter is at hand that dictates the balance between pore size and concentration of fixed charges within the membrane.

Using these capillary-OEIP devices, we have also detailed a straightforward method to characterize the PE's fixed charge concentration via an ion exchange process. Because the fixed charge concentration of a PE in its operational state directly relates to its ionic conductivity, as well as its charge selectivity, reliable methods to determine its value are important for characterizing and comparing device performance between various PEs, OEIPs, and related technologies.

Additionally, a computational physics model was employed to investigate unexpected delivery dynamics and low transport efficiency of the capillary-OEIP devices during transport and delivery of the dye molecule indigo carmine, leading to several generalizable insights into the operational characteristics of OEIP technology. These insights are particularly relevant for the transport and delivery of lower mobility ions. Importantly, our results show that the specific geometry of the PE/electrolyte interface at the OEIP outlet together with the mobility/solubility of the transported ions are of critical importance to the device's performance.

In particular, it was shown that the combination of low ionic mobility, a void in the PE close to the OEIP channel outlet, and/or high operational current can lead to an increase in ionic concentration at the PE outlet. This increased ionic concentration can cause a breakdown in the membrane's charge selectivity and results in a significant backflow of co-ions from target electrolyte toward source electrolyte. The resulting backflow of co-ions can greatly reduce the OEIP's efficiency in delivering a specific ionic species and could result in undesirable chemical interactions. For example, in the case of permselectivity failure in an acidic target medium, the negative charge of transported anions in the PE could be neutralized by a strong backflow of protons, potentially resulting in further reduced ion delivery efficiencies or blockage of the ion channel all together. Importantly, the presence of a void in a PE channel is not restricted to capillary-OEIPs and could be found in other OEIP device geometries. For example, "voids" of similar dimensions can be found in vertical OEIP structures ${ }^{15,31}$, where ion delivery occurs through patterned holes in the encapsulation layers that are typically $2-50 \mu \mathrm{m}$ thick. 
Taken together, these results lead us to several conclusions regarding the design and operation of OEIP devices, particularly for ions with low diffusion coefficients $\left(D_{\mathrm{i}}<10^{-10} \mathrm{~m}^{2} \mathrm{~s}^{-1}\right)$. Namely, there are geometrical and ion solubility adjustments that can be made to diminish the Donnan exclusion failure at the outlet. Specifically, reducing or eliminating the void region, or even extending the PE into the target electrolyte, would likely help. Alternatively, using compounds that are more soluble, or operating OEIPs with a source solvent that increases the solubility of the transported ions within the channel could potentially help reduce the degree of Donnan exclusion failure by maintaining a higher effective fixed charge concentration of the PE. It is clear from our work here that further studies, with a greater variety of ions and under a variety of experimental conditions are still needed to tease out the details of OEIP mediated delivery of "large" ions.

Overall, these considerations point to the fact that for a given device geometry and set of ions, there will be an optimum current range for achieving the best delivery efficiency. As shown above, for low mobility ions, this optimum current may be in the range of nanoamps for micron sized OEIP outlets. However, this is not likely to pose a significant hurdle in implementing capillary-OEIPs in vivo and in the clinic as even at "low" currents, these small amounts of highly concentrated bioactive compounds could significantly affect cells directly at the OEIP outlet. Furthermore, the capillary-OEIP geometry, coupled with the results and design principles outlined above provide a path forward for even further miniaturization by indicating delivery that is even more local and specific.

\section{SUPPORTING INFORMATION}

Additional details are provided for: Material Synthesis and Characterization, CapillaryOEIP Fabrication, Characterization of Capillary-OEIPs, dPG Selectivity, $\mathrm{Zn}^{2+}$ Delivery to a Plant Root, Large Ion Transport in dPGs, Measurement of Large Ion Delivery, Numerical Simulations. 


\section{AUTHOR INFORMATION}

\section{Corresponding Author}

*Magnus Berggren. Email: magnus.berggren@liu.se

\section{Present Addresses}

† Division of Micro and Nanosystems, KTH Royal Institute of Technology, Stockholm, Sweden

\section{Author Contributions}

The manuscript was written through contributions of all authors. All authors have given approval to the final version of the manuscript. $¥$ These authors contributed equally. D.J.P., E.O.G., K.T., D.T.S., M.B. designed research; D.J.P., E.O.G., A.B., I.M. performed fabrication and characterization of devices; U.L., T.A., developed AdPG polymers. T.A. performed chemical synthesis and characterization of AdPG polymers used in the manuscript; K.T. performed computational calculations; D.J.P., A.B., and K.T. analyzed the data; D.J.P., M.B., and D.T.S. wrote the paper.

\section{Author ORCID IDs}

David J. Poxson: 0000-0002-4246-8723

Erik O. Gabrielsson: 0000-0002-0302-226X

Alberto Bonisoli: 0000-0002-8736-0396

Ulrika Linderhed: 0000-0001-9605-9151

Tobias Abrahamsson: 0000-0002-3615-1850

Isabelle Matthiesen: 0000-0003-4787-7785

Klas Tybrandt: 0000-0002-9845-446X

Magnus Berggren: 0000-0001-5154-0291

Daniel T. Simon: 0000-0002-2799-3490

\section{ACKNOWLEDGMENT}

This work was primarily supported by the Swedish Foundation for Strategic Research. Additional funding was provided by the Swedish Government Strategic Research Area in Materials Science on Advanced Functional Materials at Linköping University (Faculty Grant SFO-Mat-LiU No. 200900971), the Önnesjö Foundation, and the Knut and Alice Wallenberg Foundation. We would especially like to thank Roger Gabrielsson for his valuable assistance in the development of the dendrolyte polymer material system. 


\section{REFERENCES}

(1) Berggren, M.; Richter-Dahlfors, A. Organic Bioelectronics. Adv. Mater. 2007, 19 (20), 32013213.

(2) Simon, D. T.; Gabrielsson, E. O.; Tybrandt, K.; Berggren, M. Organic Bioelectronics: Bridging the Signaling Gap between Biology and Technology. Chem. Rev. 2016, 116 (21), 1300913041. https://doi.org/10.1021/acs.chemrev.6b00146.

(3) Rivnay, J.; Owens, R. M.; Malliaras, G. G. The Rise of Organic Bioelectronics. Chem. Mater. 2014, 26 (1), 679-685. https://doi.org/10.1021/cm4022003.

(4) Someya, T.; Dodabalapur, A.; Gelperin, A.; Katz, H. E.; Bao, Z. Integration and Response of Organic Electronics with Aqueous Microfluidics. Langmuir 2002, 18 (13), 5299-5302. https://doi.org/10.1021/la020026z.

(5) Kergoat, L.; Piro, B.; Berggren, M.; Horowitz, G.; Pham, M.-C. Advances in Organic Transistor-Based Biosensors: From Organic Electrochemical Transistors to ElectrolyteGated Organic Field-Effect Transistors. Anal. Bioanal. Chem. 2012, 402 (5), 1813-1826.

(6) Torsi, L.; Dodabalapur, A. Organic Thin-Film Transistors as Plastic Analytical Sensors. Anal. Chem. 2005, 77 (19), 380 A-387 A. https://doi.org/10.1021/ac053475n.

(7) Pyo, M.; Bohn, C. C.; Smela, E.; Reynolds, J. R.; Brennan, A. B. Direct Strain Measurement of Polypyrrole Actuators Controlled by the Polymer/Gold Interface. Chem. Mater. 2003, 15 (4), 916-922. https://doi.org/10.1021/cm020312w.

(8) Saltó, C.; Saindon, E.; Bolin, M.; Kanciurzewska, A.; Fahlman, M.; Jager, E. W. H.; Tengvall, 
P.; Arenas, E.; Berggren, M. Control of Neural Stem Cell Adhesion and Density by an Electronic Polymer Surface Switch. Langmuir 2008, 24 (24), 14133-14138. https://doi.org/10.1021/la8028337.

(9) Xie, C.; Li, P.; Han, L.; Wang, Z.; Zhou, T.; Deng, W.; Wang, K.; Lu, X. Electroresponsive and Cell-Affinitive Polydopamine/Polypyrrole Composite Microcapsules with a Dual-Function of on-Demand Drug Delivery and Cell Stimulation for Electrical Therapy. NPG Asia Mater. 2017, 9 (3), e358-9. https://doi.org/10.1038/am.2017.16.

(10) Jager, E. W. H.; Smela, E.; Inganäs, O. Microfabricating Conjugated Polymer Actuators. $\begin{array}{lllll}\text { Science } & \text { (80-. } & \text { 2000, } & \end{array}$ https://doi.org/10.1126/science.290.5496.1540.

(11) Isaksson, J.; Kjäll, P.; Nilsson, D.; Robinson, N. D.; Berggren, M.; Richter-Dahlfors, A. Electronic Control of Ca2+ Signalling in Neuronal Cells Using an Organic Electronic Ion Pump. Nat. Mater. 2007, 6, 673-679. https://doi.org/10.1038/nmat1963.

(12) Tybrandt, K.; Larsson, K. C.; Kurup, S.; Simon, D. T.; Kjäll, P.; Isaksson, J.; Sandberg, M.; Jager, E. W. H.; Richter-Dahlfors, A.; Berggren, M. Translating Electronic Currents to Precise Acetylcholine-Induced Neuronal Signaling Using an Organic Electrophoretic Delivery Device. Adv. Mater. 2009, 21, 4442-4446. https://doi.org/10.1002/adma.200900187.

(13) Jonsson, A.; Song, Z.; Nilsson, D.; Meyerson, B. a.; Simon, D. T.; Linderoth, B.; Berggren, M. Therapy Using Implanted Organic Bioelectronics. Sci. Adv. 2015, 1 (4), e1500039e1500039. https://doi.org/10.1126/sciadv.1500039. 
(14) Proctor, C. M.; Slézia, A.; Kaszas, A.; Ghestem, A.; del Agua, I.; Pappa, A. M.; Bernard, C.; Williamson, A.; Malliaras, G. G. Electrophoretic Drug Delivery for Seizure Control. Sci. Adv. 2018, 4 (8). https://doi.org/10.1126/sciadv.aau1291.

(15) Jonsson, A.; Sjöström, T. A.; Tybrandt, K.; Berggren, M.; Simon, D. T. Chemical Delivery Array with Millisecond Neurotransmitter Release. Sci. Adv. 2016, 2 (11), 1-7. https://doi.org/10.1126/sciadv.1601340.

(16) Simon, D. T.; Kurup, S.; Larsson, K. C.; Hori, R.; Tybrandt, K.; Goiny, M.; Jager, E. W. H.; Berggren, M.; Canlon, B.; Richter-Dahlfors, A. Organic Electronics for Precise Delivery of Neurotransmitters to Modulate Mammalian Sensory Function. Nat. Mater. 2009, 8 (9), 742-746. https://doi.org/10.1038/nmat2494.

(17) Poxson, D. J.; Karady, M.; Gabrielsson, R.; Alkattan, A. Y.; Gustavsson, A.; Doyle, S. M.; Robert, S.; Ljung, K.; Grebe, M.; Simon, D. T.; Berggren, M. Regulating Plant Physiology with Organic Electronics. Proc. Natl. Acad. Sci. 2017, 114 (18), 4597-4602. https://doi.org/10.1073/pnas.1617758114.

(18) Keiser, G.; Xiong, F.; Cui, Y.; Shum, P. P. Review of Diverse Optical Fibers Used in Biomedical Research and Clinical Practice. J. Biomed. Opt. 2014, 19 (8), 080902. https://doi.org/10.1117/1.JBO.19.8.080902.

(19) Zhang, B.; Liu, H.; Karger, B. L.; Foret, F. Microfabricated Devices for Capillary Electrophoresis-Electrospray Mass Spectrometry. Anal. Chem. 1999, 71 (15), 3258-3264. https://doi.org/10.1021/ac990090u. 
(20) Zhang, B.; Liu, H.; Karger, B. L.; Foret, F. Microfabricated Devices for Capillary Electrophoresis-Electrospray Mass Spectrometry. Anal. Chem. 1999, 71 (15), 3258-3264. https://doi.org/10.1021/ac990090u.

(21) Palacios, I.; Block, P. C.; Brandi, S.; Blanco, P.; Casal, H.; Pulido, J.; Munoz, S.; Empaire, G. D.; Ortega, M. A.; Jacobs, M. ; Vlahakes, G. Percutaneous Balloon Valvotomy for Patients with Severe Mitral Stenosis.

(22) Arbring Sjöström, T.; Berggren, M.; Gabrielsson, E. O.; Janson, P.; Poxson, D. J.; Seitanidou, M.; Simon, D. T. A Decade of Iontronic Delivery Devices. Adv. Mater. Technol. 2018, 3 (5). https://doi.org/10.1002/admt.201700360.

(23) Kontturi, K.; Murtomäki, L.; Manzanares, J. Ionic Transport Processes: In Electrochemistry and Membrane Science: In Electrochemistry and Membrane Science; Oxford University Press, 2008.

(24) Tybrandt, K.; Forchheimer, R.; Berggren, M. Logic Gates Based on Ion Transistors. Nat. Commun. 2012, 3 (May), 871. https://doi.org/10.1038/ncomms1869.

(25) Chun, H. Development of a Low Flow-Resistive Charged Nanoporous Membrane in a Microchip for Fast Electropreconcentration. Electrophoresis 2018, 39 (17), 2181-2187. https://doi.org/10.1002/elps.201800093.

(26) Shin, I. H.; Kim, K. J.; Kim, J.; Kim, H. C.; Chun, H. Cation-Selective Electropreconcentration. Lab Chip 2014, 14 (11), 1811-1815. https://doi.org/10.1039/c4lc00024b.

(27) Chun, H.; Chung, T. D.; Ramsey, J. M. High Yield Sample Preconcentration Using a Highly 
Ion-Conductive Charge-Selective Polymer. Anal. Chem. 2010, 82 (14), 6287-6292. https://doi.org/10.1021/ac101297t.

(28) Han, J. H.; Kim, K. B.; Kim, H. C.; Chung, T. D. Ionic Circuits Based on Poly Electrolyte Diodes on a Microchip. Angew. Chemie - Int. Ed. 2009, 48 (21), 3830-3833. https://doi.org/10.1002/anie.200900045.

(29) Cornet, N.; Diat, O.; Gebel, G.; Jousse, F.; Marsacq, D.; Mercier, R.; Pineri, M. Sulfonated Polyimide Membranes: A New Type of Ion-Conducting Membrane for Electrochemical Applications. J. New Mater. Electrochem. Syst. 2000, 3 (1), 33-42.

(30) Boon, N.; De La Cruz, M. O. "Soft" Amplifier Circuits Based on Field-Effect lonic Transistors. Soft Matter 2015, 11 (24), 4793-4798. https://doi.org/10.1039/c5sm00573f.

(31) Tybrandt, K. Exploring the Potential of Ionic Bipolar Diodes for Chemical Neural Interfaces. Soft Matter 2017, 13 (44), 8171-8177. https://doi.org/10.1039/C7SM01732D.

(32) Volkov, A. V.; Tybrandt, K.; Berggren, M.; Zozoulenko, I. V. Modeling of Charge Transport in Ion Bipolar Junction Transistors. Langmuir 2014, 30 (23), 6999-7005. https://doi.org/10.1021/la404296g.

(33) Zhang, S.; Kumar, P.; Nouas, A. S.; Fontaine, L.; Tang, H.; Cicoira, F. Solvent-Induced Changes in PEDOT:PSS Films for Organic Electrochemical Transistors. APL Mater. 2015, 3 (1), 1-8. https://doi.org/10.1063/1.4905154.

(34) Mariame, C.; Drissa, B.; Ellogne, Z. Study of Junction Carbon Paste Electrode / Indigo Carmine / Mercury ( II ): Application of the Law Cottrell. 2016, 5 (1), 1300-1305. 
For Table of Contents Only

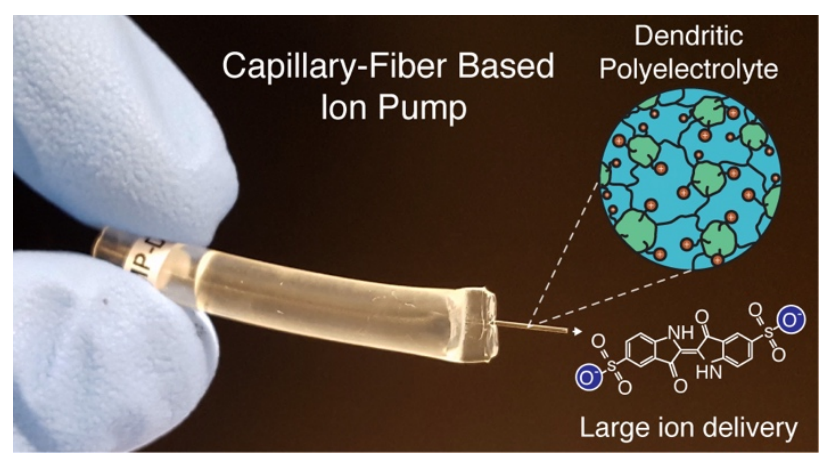

